\title{
NOR gate response in a double quantum ring: An exact result
}

\author{
Santanu K. Maiti ${ }^{1,2, *}$ \\ ${ }^{1}$ Theoretical Condensed Matter Physics Division, Saha Institute of Nuclear Physics, \\ 1/AF, Bidhannagar, Kolkata-700 064, India \\ ${ }^{2}$ Department of Physics, Narasinha Dutt College, 129, Belilious Road, Howrah-711 101, India
}

\begin{abstract}
NOR gate response in a double quantum ring, where each ring is threaded by a magnetic flux $\phi$, is investigated. The double quantum ring is sandwiched symmetrically between two semi-infinite onedimensional metallic electrodes and two gate voltages, namely, $V_{a}$ and $V_{b}$, are applied, respectively, in lower arms of the two rings those are treated as the two inputs of the NOR gate. A simple tightbinding model is used to describe the system and all the calculations are done through the Green's function formalism. Here we exactly calculate the conductance-energy and current-voltage characteristics as functions of the ring-to-electrode coupling strengths, magnetic flux and gate voltages. Our numerical study predicts that, for a typical value of the magnetic flux $\phi=\phi_{0} / 2\left(\phi_{0}=c h / e\right.$, the elementary fluxquantum), a high output current (1) (in the logical sense) appears if both the inputs to the gate are low $(0)$, while if one or both are high (1), a low output current (0) results. It clearly demonstrates the NOR gate behavior and this aspect may be utilized in designing an electronic logic gate.
\end{abstract}

PACS No.: 73.23.-b; 73.63.Rt.

Keywords: A. Double quantum ring; D. Conductance; D. $I-V$ characteristic; D. NOR gate.

*Corresponding Author: Santanu K. Maiti

Electronic mail: santanu.maiti@saha.ac.in 


\section{Introduction}

Low dimensional model quantum systems like, quantum rings, quantum dots, arrays of quantum rings and dots, etc, have been the objects of intense research, both in theory and in experiments, mainly due to the fact that these simple looking systems are prospective candidates for nano devices in electronic as well as spintronic engineering. The key idea of manufacturing nano devices is based on the concept of quantum interference effect, and it is generally preserved in the samples of much smaller sizes. While, the effect disappears for larger systems. A mesoscopic metallic ring is a promising example where electronic motion is confined, and for small enough in size, electron transport becomes phase-coherent throughout the ring [1. With the aid of two such metallic rings, we construct a double quantum ring, and, here we will explore how such a simple geometric model can be used in designing an electronic NOR gate. To reveal this fact, we make a bridge system by inserting the double quantum ring between two external electrodes, namely, source and drain (see Fig. 1). This is the so-called source-double quantum ringdrain bridge. The theoretical description of electron transfer in a bridge system has got much progress following the pioneering work of Aviram and Ratner [2]. Later, several excellent experiments [3, 4, 5] have been done in different bridge systems to understand the basic mechanisms underlying the electron transport. Though in literature many theoretical [6, 17, 8, 9, 10, 11, 12, 13, 14, 15, 16, 17, 18, 19, 20. as well as experimental works [3, 4, 5, on electron transport are available, yet lot of controversies are still present between the theory and experiment, and the complete knowledge of the conduction mechanism in this scale is not very well established even today.

The main focus of the present work is to describe the NOR gate response in a double quantum ring where each ring is threaded by a magnetic flux $\phi$. The double quantum ring is sandwiched symmetrically between the electrodes, and the lower arms of the ring are subjected to the gate voltages $V_{a}$ and $V_{b}$, respectively, those are considered as the two inputs of the NOR gate (see Fig. 11). Here we use a simple tight-binding model to describe the system and we perform all the calculations numerically. The NOR gate behavior is addressed by studying the conductance-energy and current-voltage characteristics in terms of the ring-to-electrode coupling strengths, magnetic flux and gate voltages. Our nu- merical results propose that for a typical value of the magnetic flux, $\phi=\phi_{0} / 2$, a high output current (1) (in the logical sense) is available only when both the two inputs to the gate are low (0). While if anyone or both are high (1), a low output current (0) results. This feature clearly demonstrates the NOR gate behavior and it may be utilized in manufacturing an electronic logic gate. To the best of our knowledge the NOR gate response in such a simple system has not been described earlier in the literature.

The paper is arranged as follow. Following the introduction (Section 1), in Section 2, we present the model and the theoretical formulations for our calculations. Section 3 discusses the significant results, and finally, we summarize our results in Section 4.

\section{Model and the synopsis of the theoretical background}

We begin by referring to Fig. 1 A double quantum ring is sandwiched symmetrically between two semiinfinite one-dimensional (1D) metallic electrodes. Each ring is threaded by a magnetic flux $\phi$, the so-called Aharonov-Bohm (AB) flux which is the key controlling parameter for the whole operation of the NOR gate. The atomic sites $a$ and $b$ in the lower arms of the two rings are subjected to the gate voltages $V_{a}$ and $V_{b}$ through the gate electrodes gate-a and gate-b, respectively. These gate voltages are variable and treated as the two inputs of the NOR gate. In a similar way we also apply two other gate voltages $V_{c}$ and $V_{d}$, those are not varying, in the atomic sites $c$ and $d$ in the upper arms of the two rings via the gate electrodes gate-c and gate-d, respectively. All these gate electrodes are ideally isolated from the rings, and here we assume that the gate voltages each operate on the atomic sites nearest to the plates only. While, in complicated geometric models, the effect must be taken into account for the other atomic sites, though the effect becomes too small. The actual scheme of connections with the batteries for the operation of the NOR gate is clearly presented in the figure (Fig. (1), where the source and the gate voltages are applied with respect to the drain.

Based on the Landauer conductance formula 21, 22 we determine the conductance $(g)$ of the double quantum ring. At much low temperatures and bias voltage it can be expressed in the form,

$$
g=\frac{2 e^{2}}{h} T
$$


where $T$ gives the transmission probability of an electron across the double quantum ring. In terms of the Green's function of the double quantum ring and its coupling to the electrodes, the transmission probability can be represented by the relation [21. 22 ,

$$
T=\operatorname{Tr}\left[\Gamma_{S} G_{R}^{r} \Gamma_{D} G_{R}^{a}\right]
$$

where $G_{R}^{r}$ and $G_{R}^{a}$ are respectively the retarded and advanced Green's functions of the double quantum ring including the effects of the electrodes. The parameters $\Gamma_{S}$ and $\Gamma_{D}$ describe the coupling of the

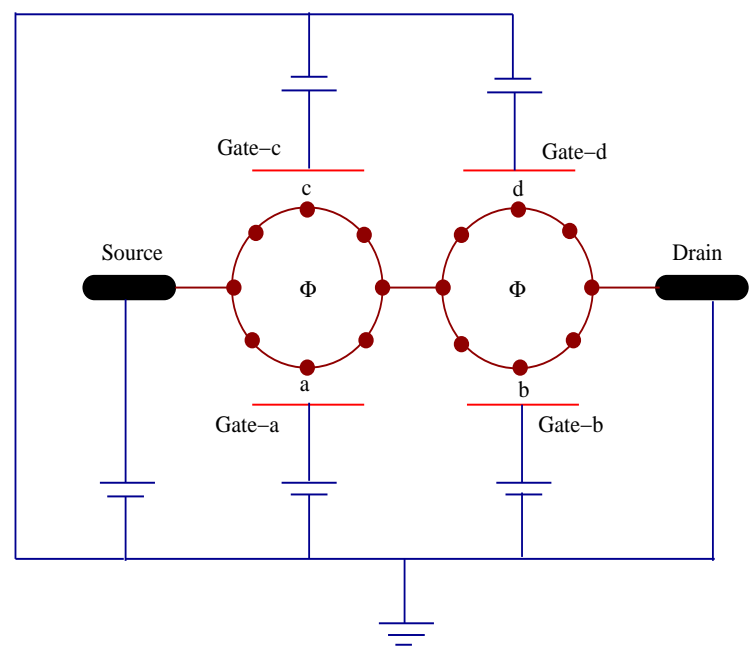

Figure 1: (Color online). The scheme of connections with the batteries for the operation of the NOR gate.

double quantum ring to the source and drain, respectively. For the complete system i.e., the double quantum ring, source and drain, the Green's function is defined as,

$$
G=(E-H)^{-1}
$$

where $E$ is the injecting energy of the source electron. In order to evaluate this Green's function, the inversion of an infinite matrix is required since the complete system consists of the finite size double quantum ring and the two semi-infinite metallic electrodes. However, the entire system can be divided into sub-matrices corresponding to the individual sub-systems and the effective Green's function for the double quantum ring can be written as,

$$
G_{R}=\left(E-H_{R}-\Sigma_{S}-\Sigma_{D}\right)^{-1}
$$

where $H_{R}$ is the Hamiltonian of the double quantum ring. Withing the approximation of the non- interacting picture, the Hamiltonian can be expressed as,

$$
\begin{aligned}
H_{R}= & \sum_{i}\left(\epsilon_{i}+V_{a} \delta_{i a}+V_{b} \delta_{i b}+V_{c} \delta_{i c}+V_{d} \delta_{i d}\right) \\
& c_{i}^{\dagger} c_{i}+\sum_{<i j>} t\left(c_{i}^{\dagger} c_{j} e^{i \theta}+c_{j}^{\dagger} c_{i} e^{-i \theta}\right)
\end{aligned}
$$

In this Hamiltonian, $\epsilon_{i}$ 's are the site energies for all the sites $i$, except the sites $i=a, b, c$ and $d$ where the gate voltages $V_{a}, V_{b}, V_{c}$ and $V_{d}$ are applied. These gate voltages can be incorporated through the site energies as expressed in the above Hamiltonian. $c_{i}^{\dagger}\left(c_{i}\right)$ is the creation (annihilation) operator of an electron at the site $i$ and $t$ is the hopping integral among the two neighboring sites in each ring. The hopping strength between the two sites through which the rings are coupled to each other is also set to $t$, for the sake of simplicity. The phase factor $\theta$ follows the relation, $\theta=2 \pi \phi / N \phi_{0}$, which comes due to the penetration of the $\mathrm{AB}$ flux $\phi$ through the ring, where $N$ represents the total number of atomic sites (filled red circles) in each ring. The effect of the magnetic flux $\phi$ enters explicitly into the above Hamiltonian (Eq. (5) ), and since no magnetic field is penetrated anywhere in the circumferences of the two rings, the above Hamiltonian is free from any Zeeman term. A similar kind of tightbinding Hamiltonian is also taken into account, except the phase factor $\theta$, to describe the semi-infinite 1D metallic electrodes where the Hamiltonian is parametrized by constant on-site potential $\epsilon^{\prime}$ and nearest-neighbor hopping integral $t^{\prime}$. The double quantum ring is coupled to the electrodes by the parameters $\tau_{S}$ and $\tau_{D}$, those correspond to the coupling strengths to the source and drain, respectively, and they (coupling parameters) enter into the terms $\Sigma_{S}$ and $\Sigma_{D}$ (see Eq. (44) 21. These factors $\left(\Sigma_{S}\right.$ and $\left.\Sigma_{D}\right)$ represent the self-energies due to the coupling of the double quantum ring to the source and drain, respectively, where all the information of the coupling are included into these two self-energies. The detailed description is available in the reference [21.

The current $I$ passing through the double quantum ring can be expressed in terms of the applied bias voltage $V$ by the relation [21,

$$
I(V)=\frac{e}{\pi \hbar} \int_{E_{F}-e V / 2}^{E_{F}+e V / 2} T(E) d E
$$

where $E_{F}$ is the equilibrium Fermi energy. Here we make a realistic assumption that the entire voltage is dropped across the ring-electrode interfaces, and 
it is examined that under such an assumption the $I-V$ characteristics do not change their qualitative features.

In this work, all the results are computed only at absolute zero temperature. These results are also valid even for some finite (low) temperatures, since the broadening of the energy levels of the double quantum ring due to its coupling to the electrodes becomes much larger than that of the thermal broadening [21]. On the other hand, at high temperature limit, all these features completely disappear. This is due to the fact that the phase coherent length decreases significantly with the rise of temperature where the contribution comes mainly from the scattering on phonons, and therefore, the quantum interference effect vanishes. For the sake of simplicity, we take the unit $c=e=h=1$ in our present study.

\section{Results and discussion}

Let us start our discussion by mentioning the values of the different parameters associated with the numerical calculations. In the double quantum ring,
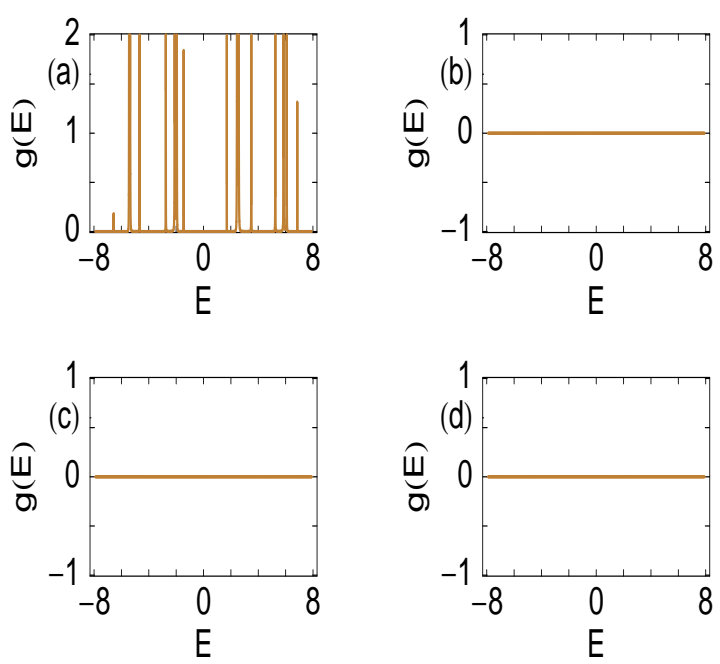

Figure 2: (Color online). $g$ - $E$ spectra for a double quantum ring with $M=16, V_{c}=V_{d}=2$ and $\phi=$ 0.5 in the limit of weak-coupling. (a) $V_{a}=V_{b}=0$, (b) $V_{a}=2$ and $V_{b}=0$, (c) $V_{a}=0$ and $V_{b}=2$ and (d) $V_{a}=V_{b}=2$.

the on-site energy $\epsilon_{i}$ is set at 0 for all the atomic sites $i$, except the sites $a, b, c$ and $d$, where the site energies are considered as $V_{a}, V_{b}, V_{c}$ and $V_{d}$, respectively, and the nearest-neighbor hopping strength $t$ is fixed at 3. On the other hand, for the two side attached 1D metallic electrodes the on-site energy $\left(\epsilon^{\prime}\right)$ and the nearest-neighbor hopping integral $\left(t^{\prime}\right)$ are chosen as 0 and 4 , respectively. The gate voltages $V_{c}$ and $V_{d}$, those are not varying, are fixed at the value 2 i.e., $V_{c}=V_{d}=2$, and the Fermi energy $E_{F}$ is set at 0 . Throughout the discussion, we focus all the essential features of electron transport for the two limiting cases depending on the strength of the coupling of the double quantum ring to the source and drain. Case $I$ : The weak-coupling limit. It is specified by the condition $\tau_{S(D)}<<t$. For this regime we choose $\tau_{S}=\tau_{D}=0.5$. Case $I I$ : The strongcoupling limit. This is mentioned by the condition $\tau_{S(D)} \sim t$. In this particular regime, we set the values of the parameters as $\tau_{S}=\tau_{D}=2.5$. The key
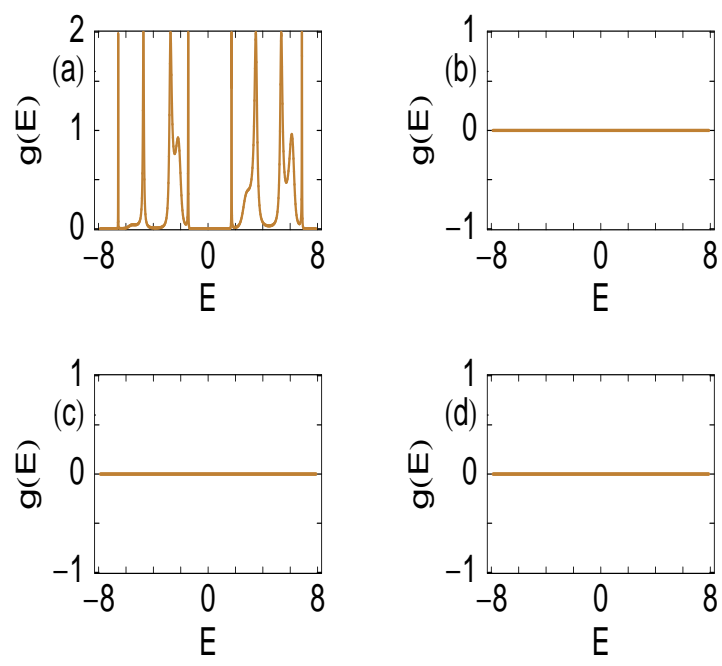

Figure 3: (Color online). $g$ - $E$ spectra for a double quantum ring with $M=16, V_{c}=V_{d}=2$ and $\phi=$ 0.5 in the limit of strong-coupling. (a) $V_{a}=V_{b}=0$, (b) $V_{a}=2$ and $V_{b}=0$, (c) $V_{a}=0$ and $V_{b}=2$ and (d) $V_{a}=V_{b}=2$.

controlling parameter for all these calculations is the $\mathrm{AB}$ flux $\phi$, threaded by each ring, which is fixed at $\phi_{0} / 2$ i.e., 0.5 in our chosen unit $c=e=h=1$.

As representative examples, in Fig. 2 we display the variation of the conductance $g$ as a function of the injecting electron energy $E$ for a double quantum ring with $M=16(M=2 N$, the total number of atomic sites in the double quantum ring, since each ring contains $N$ atomic sites) in the weakcoupling limit, where (a), (b), (c) and (d) represent the results for the four different cases of the gate voltages $V_{a}$ and $V_{b}$, respectively. Quite interestingly from these spectra we observe that, for the 
case when both the two inputs $V_{a}$ and $V_{b}$ are identical to 2 i.e., both are high, the conductance $g$ becomes exactly zero for the full range of the energy $E$ (see Fig. 2(d)). The exactly similar response is also visible for the two other cases where anyone of the two inputs is high and other is low. The results are shown in Figs. 2(b) and (c), respectively. Hence, for all these three cases (Figs. 2(b)-(d)), no electron conduction takes place from the source to the drain through the double quantum ring. The electron conduction through the bridge system is allowed only for the typical case where both the two inputs two the gates are low i.e., $V_{a}=V_{b}=0$. The spectrum is given in Fig. 2(a). It is noticed that, for some particular energies the conductance exhibits sharp resonant peaks. At these resonant energies, the conductance approaches the value 2 , and ac-
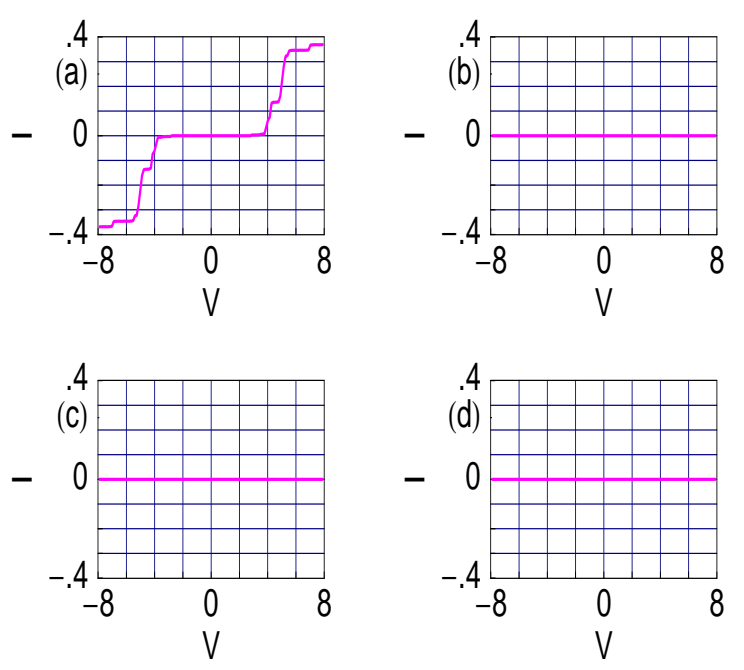

Figure 4: (Color online). Current $I$ as a function of the applied bias voltage $V$ for a double quantum ring with $M=16, V_{c}=V_{d}=2$ and $\phi=0.5$ in the weak-coupling limit. (a) $V_{a}=V_{b}=0$, (b) $V_{a}=2$ and $V_{b}=0$, (c) $V_{a}=0$ and $V_{b}=2$ and (d) $V_{a}=$ $V_{b}=2$.

cordingly, the transmission probability $T$ goes to unity, since the relation $g=2 T$ holds from the Landauer conductance formula (see Eq. (1) with $e=h=1)$. All these resonant peaks are associated with the energy eigenvalues of the double quantum ring, and therefore, it can be emphasized that the conductance spectrum manifests itself the electronic structure of the double quantum ring. Now we try to explain the roles of the gate voltages on the electron transport in these four different cases. The probability amplitude of getting an electron from the source to drain across the double quantum ring depends on the combined effect of the quantum interferences of the electronic waves passing through the upper and lower arms of the two rings. For a symmetrically connected ring (lengths of the two arms of the ring are identical to each other), threaded by an AB flux $\phi$, the probability amplitude of getting an electron across the ring becomes exactly zero $(T=0)$ for the typical flux, $\phi=\phi_{0} / 2$. This is due to the result of the quantum interference among the two waves in the two arms of the ring [1], which can be shown through few simple mathematical steps. Thus for the particular case when both the two inputs to the gate are high i.e., $V_{a}=V_{b}=2$, the upper and lower arms of the two rings become exactly identical since the gate voltages $V_{c}$ and $V_{d}$ in the upper arms are also fixed at the value 2 . This provides the vanishing transmission probability. If the input voltages $V_{a}$ and $V_{b}$ are different from the potential applied in the atomic sites $c$ and $d$, then the upper and the lower arms of the two rings are no longer identical to each other and the transmission probability will not vanish. Thus, to get the zero transmission probability when the inputs are

Table 1: NOR gate response in the limit of weakcoupling. The current $I$ is computed at the bias voltage 6.02 .

\begin{tabular}{|c|c|c|}
\hline \hline Input-I $\left(V_{a}\right)$ & Input-II $\left(V_{b}\right)$ & Current $(I)$ \\
\hline 0 & 0 & 0.346 \\
\hline 2 & 0 & 0 \\
\hline 0 & 2 & 0 \\
\hline 2 & 2 & 0 \\
\hline \hline
\end{tabular}

high, we should tune $V_{c}$ and $V_{d}$ properly, observing the input potentials and vice versa. The similar behavior is also noticed for the two other cases $\left(V_{a}=2, V_{b}=0\right.$ and $\left.V_{a}=0, V_{b}=2\right)$, where the symmetry is broken in only one ring out of these two by making the gate voltage either in the site $b$ or in $a$ to zero, maintaining the symmetry in the other ring. The reason is that, when anyone of the two gates $\left(V_{a}\right.$ and $\left.V_{b}\right)$ becomes zero, the symmetry between the upper and lower arms is broken only in one ring which provides non-zero transmission probability across the ring. While, for the other ring where the gate voltage is applied, the symmetry between the two arms becomes preserved which gives zero transmission probability. Accordingly, the combined effect of these two rings provides van- 
ishing transmission probability across the bridge, as the two rings are coupled to each other. The non-zero value of the transmission probability appears only when the symmetries of both the two rings are identically broken, and it is available for the particular case when both the two inputs to the gate are low i.e., $V_{a}=V_{b}=0$. This feature clearly demonstrates the NOR gate behavior. With these properties, we get additional one feature when the coupling strength of the double quantum ring to the electrodes is increased from the low regime to the high one. In the limit of strong-coupling, all these resonant peaks get substantial widths compared to the weak-coupling limit. The results are shown in
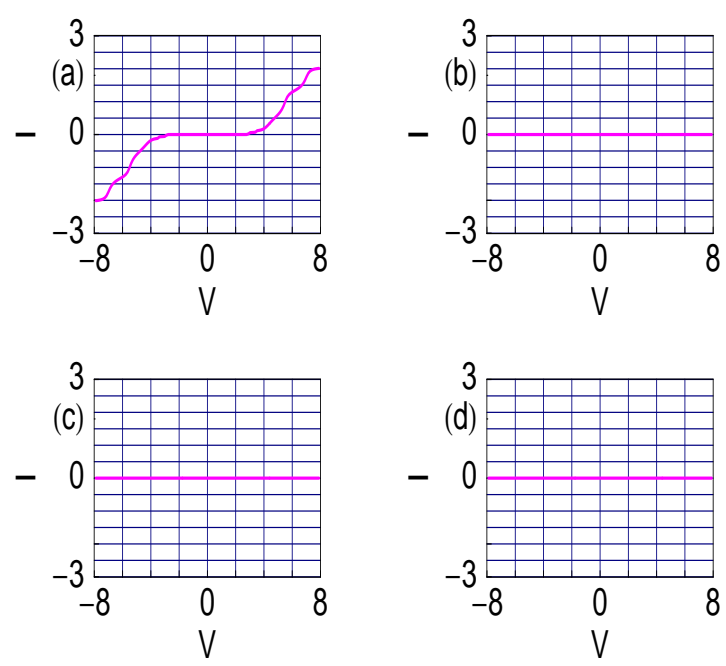

Figure 5: (Color online). Current $I$ as a function of the applied bias voltage $V$ for a double quantum ring with $M=16, V_{c}=V_{d}=2$ and $\phi=0.5$ in the strong-coupling limit. (a) $V_{a}=V_{b}=0$, (b) $V_{a}=2$ and $V_{b}=0$, (c) $V_{a}=0$ and $V_{b}=2$ and (d) $V_{a}=V_{b}=2$.

Fig. 3. where all the other parameters are identical to those in Fig. 2. The contribution for the broadening of the resonant peaks in this strong-coupling limit appears from the imaginary parts of the selfenergies $\Sigma_{S}$ and $\Sigma_{D}$, respectively [21. Hence, by tuning the coupling strength, we can get the electron transmission across the double quantum ring for the wider range of energies and it provides an important signature in the study of the currentvoltage $(I-V)$ characteristics.

All these features of electron transfer become much more clearly visible by studying the $I-V$ characteristics. The current passing through the double quantum ring is computed from the integration pro- cedure of the transmission function $T$ as prescribed in Eq. (6). The transmission function varies exactly similar to that of the conductance spectrum, differ only in magnitude by the factor 2 since the relation $g=2 T$ holds from the Landauer conductance formula Eq. (11). As illustrative examples, in Fig. 4 we show the variation of the current $I$ as a function of the applied bias voltage $V$ for a double quantum ring with $M=16$ in the limit of weak-coupling, where (a), (b), (c) and (d) correspond to the results for the different cases of the two input voltages, respectively. For the cases when either both the two inputs to the gate are high $\left(V_{a}=V_{b}=2\right)$, or anyone of the two inputs is high and other is low $\left(V_{a}=2\right.$, $V_{b}=0$ or $\left.V_{a}=0, V_{b}=2\right)$, the current drops exactly to zero for the whole range of the bias voltage. The results are shown in Figs. 4)(b)-(d), and, the vanishing behavior of the current in these three different cases can be clearly understood from the conductance spectra given in Figs. 2(b)-(d), since the current is computed from the integration procedure of the transmission function $T$. The finite

Table 2: NOR gate response in the limit of strongcoupling. The current $I$ is computed at the bias voltage 6.02 .

\begin{tabular}{|c|c|c|}
\hline \hline Input-I $\left(V_{a}\right)$ & Input-II $\left(V_{b}\right)$ & Current $(I)$ \\
\hline 0 & 0 & 1.295 \\
\hline 2 & 0 & 0 \\
\hline 0 & 2 & 0 \\
\hline 2 & 2 & 0 \\
\hline \hline
\end{tabular}

value of the current is observed only for the typical case where both the two inputs to the gate are low i.e., $V_{a}=V_{b}=0$. The result is shown in Fig. 4(a). From this figure it is observed that the current exhibits staircase-like structure with fine steps as a function of the applied bias voltage. This is due to the existence of the sharp resonant peaks in the conductance spectrum in the weak-coupling limit, since the current is computed by the integration method of the transmission function $T$. With the increase of the bias voltage $V$, the electrochemical potentials on the electrodes are shifted gradually, and finally cross one of the quantized energy levels of the double quantum ring. Therefore, a current channel is opened up which produces a jump in the $I-V$ spectrum. Addition to these behaviors, it is also important to note that the non-zero value of the current appears beyond a finite value of $V$, the 
so-called threshold voltage $\left(V_{t h}\right)$. This $V_{t h}$ can be tuned by controlling the size $(N)$ of the two rings. From these $I-V$ spectra, the behavior of the NOR gate response is clearly observed. To make it more clearer, in Table 1 we present a quantitative estimate of the typical current amplitude, computed at the bias voltage $V=6.02$, in this weak-coupling limit. It shows $I=0.346$ only when both the two inputs to the gate are low $\left(V_{a}=V_{b}=0\right)$, while for the other three cases when either $V_{a}=V_{b}=2$ or $V_{a}=2, V_{b}=0$ or $V_{a}=0, V_{b}=2$, the current $I$ gets the value 0 . In the same footing, as above, here we also discuss the $I-V$ characteristics in the limit of strong-coupling. In this limit, the current varies almost continuously with the applied bias voltage and achieves much larger amplitude than the weakcoupling case (Fig. 4), as presented in Fig. 5. The reason is that, in the limit of strong-coupling all the resonant peaks get broadened which provide larger current in the integration procedure of the transmission function $T$. Thus by tuning the strength of the ring-to-electrodes coupling, we can achieve very large current amplitude from the very low one for the same bias voltage $V$. All the other properties i.e., the dependences of the gate voltages on the $I-V$ characteristics are exactly similar to those as given in Fig. 4. In this strong-coupling limit we also make a quantitative study for the typical current amplitude, given in Table 2 where the current amplitude is determined at the same bias voltage $(V=6.02)$ as earlier. The response of the output current is exactly similar to that as given in Table 1 Here the non-zero value of the current gets the value 1.295 which is much larger compared to the weakcoupling case that provides the value 0.346. From these results the NOR gate response in a double quantum ring can be clearly manifested.

\section{Concluding remarks}

In conclusion, we have explored the NOR gate response in a double quantum ring where each ring is threaded by a magnetic flux $\phi$. The double quantum ring is attached symmetrically to two semiinfinite 1D metallic electrodes, and two gate voltages, namely, $V_{a}$ and $V_{b}$, are applied, respectively, in the lower arms of the two rings and they are treated as the two inputs of the NOR gate. The model is described by the tight-binding Hamiltonian, and all the calculations are performed within the Green's function formalism. We have done exact numerical calculation to determine the conductance-energy and current-voltage characteristics as functions of the ring-electrode coupling strengths, magnetic flux and gate voltages. Very nicely we have noticed that, for the half flux-quantum value of $\phi\left(\phi=\phi_{0} / 2\right)$, a high output current (1) (in the logical sense) appears if both the two inputs to the gate are low (0). While, if one or both are high (1), a low output current (0) results. It clearly manifests the NOR gate response and this aspect may be utilized in designing a tailor made electronic logic gate.

Throughout our presentation, we have addressed the conductance-energy and current-voltage characteristics for a double quantum ring with total number of atomic sites $M=16$. In our model calculations, this typical number $(M=16)$ is chosen only for the sake of simplicity. Though the results presented here change numerically with the ring size $(N)$, but all the basic features remain exactly invariant. To be more specific, it is important to note that, in real situation the experimentally achievable rings have typical diameters within the range 0.4 $0.6 \mu \mathrm{m}$.

In the present work we have done all the calculations by ignoring the effects of the temperature, electron-electron correlation, disorder, etc. Due to these factors, any scattering process that appears in the arms of the rings would have influence on electronic phases, and, in consequences can disturb the quantum interference effects. Here we have assumed that in our sample all these effects are too small, and accordingly, we have neglected all these factors in this particular study.

The importance of this article is mainly concerned with (i) the simplicity of the geometry and (ii) the smallness of the size. To the best of our knowledge the NOR gate response in such a simple low-dimensional system that can be operated even at finite temperatures (low) has not been addressed earlier in the literature.

At the end, here we have designed a NOR gate using mesoscopic rings, based on the effect of quantum interference, which is a classical logic gate. On the other hand, quantum logic gates using such rings have already been proposed earlier which can be available in the reference 23 .

\section{References}

[1] R. A. Webb, S. Washburn, C. P. Umbach, R. B. Laibowitz, Phys. Rev. Lett. 54 (1985) 2696.

[2] A. Aviram, M. Ratner, Chem. Phys. Lett. 29 (1974) 277. 
[3] T. Dadosh, Y. Gordin, R. Krahne, I. Khivrich, D. Mahalu, V. Frydman, J. Sperling, A. Yacoby, I. Bar-Joseph, Nature 436 (2005) 677.

[4] J. Chen, M. A. Reed, A. M. Rawlett, J. M. Tour, Science 286 (1999) 1550.

[5] M. A. Reed, C. Zhou, C. J. Muller, T. P. Burgin, J. M. Tour, Science 278 (1997) 252.

[6] P. A. Orellana, M. L. Ladron de Guevara, M. Pacheco, A. Latge, Phys. Rev. B 68 (2003) 195321.

[7] P. A. Orellana, F. Dominguez-Adame, I. Gomez, M. L. Ladron de Guevara, Phys. Rev. B 67 (2003) 085321.

[8] A. Nitzan, Annu. Rev. Phys. Chem. 52 (2001) 681.

[9] A. Nitzan, M. A. Ratner, Science 300 (2003) 1384.

[10] D. M. Newns, Phys. Rev. 178 (1969) 1123.

[11] V. Mujica, M. Kemp, M. A. Ratner, J. Chem. Phys. 101 (1994) 6849.

[12] V. Mujica, M. Kemp, A. E. Roitberg, M. A. Ratner, J. Chem. Phys. 104 (1996) 7296.

[13] K. Walczak, Phys. Stat. Sol. (b) 241 (2004) 2555 .

[14] K. Walczak, arXiv:0309666.

[15] W. Y. Cui, S. Z. Wu, G. Jin, X. Zhao, Y. Q. Ma, Eur. Phys. J. B. 59 (2007) 47.

[16] R. Baer, D. Neuhauser, J. Am. Chem. Soc. 124 (2002) 4200.

[17] D. Walter, D. Neuhauser, R. Baer, Chem. Phys. 299 (2004) 139.

[18] K. Tagami, L. Wang, M. Tsukada, Nano Lett. 4 (2004) 209.

[19] K. Walczak, Cent. Eur. J. Chem. 2 (2004) 524.

[20] R. Baer, D. Neuhauser, Chem. Phys. 281 (2002) 353.

[21] S. Datta, Electronic transport in mesoscopic systems, Cambridge University Press, Cambridge (1997).

[22] M. B. Nardelli, Phys. Rev. B 60 (1999) 7828.

[23] P. Földi, B. Molnar, M. G. Benedict, F. M. Peeters, Phys. Rev. B 71 (2005) 033309. 